\title{
Asymptotically Optimal Scheduling for Compute-and-Forward
}

\author{
Ori Shmuel, Asaf Cohen, Omer Gurewitz \\ Ben-Gurion University of the Negev, \\ \{shmuelor, coasaf, gurewitz\}@bgu.ac.il
}

\begin{abstract}
Consider a Compute and Forward (CF) relay network with $L$ users and a single relay. The relay tries to decode a linear function of the transmitted signals. For such a network, letting all $L$ users transmit simultaneously, especially when $L$ is large, causes a significant degradation in the rate in which the relay is able to decode. In fact, the rate goes to zero very fast with $L$. Therefore, in each transmission phase only a fixed number of users should transmit, i.e., users should be scheduled.

In this work, we examine the problem of scheduling for $\mathrm{CF}$ and lay the foundations for identifying the optimal schedule which, to date, lacks a clear understanding. Specifically, we start with insights why when the number of users is large, good scheduling opportunities can be found. Then, we provide an asymptotically optimal, polynomial time scheduling algorithm and analyze it's performance. We conclude that scheduling under CF provides a gain in the system sum-rate, up to the optimal scaling law of $O(\log \log L)$.
\end{abstract}

\section{INTRODUCTION}

Compute and Forward (CF) is a coding strategy [1], introduced for relay systems consisting of multiple transmitters and multiple relays. In this scheme, the relays (receivers) decode a linear function of the received messages instead of decoding them individually. Therefore, it is a powerful technique for mitigating users' interferences, which is a prominent problem in today's wireless communication systems. The ability to exploit simultaneous transmissions is possible due to the usage of lattice codes, which enable the function of the transmitted messages to be decoded as a legitimate message. Accordingly, in recent years, the main ideas of CF were used to attain new results for linear receivers [2], the Multiple Access Channel sum capacity [3] and more [4], [5].

The performance of $\mathrm{CF}$ in the regime of large number of users and a fixed number of relays was investigated in [6]. It was shown that the CF scheme degenerates fast when the number of transmitters grows, in the sense that the relays prefer to decode a single message instead of any other linear combination of the messages. This is due to the "self" noise added to the decoding process, which tries to approximate the real channel vector with an integer coefficients vector. Specifically, as the number of simultaneously transmitting users grows, a receiver essentially prefers to treat all messages as noise apart from the message it tries to decode. As a consequence, the system's sum-rate goes to zero (even for a

This research was partially supported by European Unions Horizon 2020 Research and Innovation Program SUPERFLUIDITY, Grant Agreement 671566. moderate number of users) and in order to have any guarantee for non-zero rate, user scheduling must be applied. Namely, applying CF on large scale relaying systems, where there is a fixed number of relays, without a restriction on the number of transmitting users, would be futile.

On the other hand, restricting the number of transmitting users can provide coding opportunities for the CF scheduler, by scheduling users with channel conditions which are more favorable for $\mathrm{CF}$ while grouped together, providing gain to the overall system's sum-rate. A related improvement, while scheduling in CF networks, was presented also in [7]. Therein, the authors showed by simulation that even a simple scheduling scheme can be useful. However, no performance guarantees or analysis for the optimal schedule were carried out. In this work, we examine user scheduling in the context of $\mathrm{CF}$ schemes and explore the scheduling considerations a CF scheduler should take. Specifically, as CF relies on the appropriate match between the fading coefficients of the transmitted signals and a certain linear function with integer coefficients, with scheduling, one can influence not only the signals which participate, but also the proper choice of the function.

Main contributions: We consider a simple relay network with a single relay and $L$ transmitters where, due to the necessity for restricting the number of simultaneously transmitting users, we schedule $k$ users for transmission. This setting is sufficient to attain important results and insights for scheduling in $\mathrm{CF}$, and build the first steps for comprehending what is the optimal schedule for CF networks.

We begin with the analysis of the optimal schedule and present an asymptotically optimal, polynomial time scheduling algorithm for $\mathrm{CF}$, which maximizes the system sum-rate. The algorithm is analyzed, and its performance is lower and upper bounded. The lower bound is derived using probabilistic arguments on the properties of the optimal schedule and the upper bound is derived using a universal upper bound on the performances of CF. We show that both the lower bound and the upper bound scale as $O(\log \log L)$, which essentially proves the optimality of the suggested algorithm and the specific schedule it provides. Consequently, we are able to provide an important property of the optimal schedule; the scheduler will seek groups of users which best match a fixed, non-trivial yet deterministic coefficients vector. Therefore, we show that the gain arises solely from the proper choice of users for that vector, and not from actually optimizing on the 
coefficients vector, like CF suggests for finite systems.

\section{System Model And KnOWn Results}

Consider a multi-user, single-relay network, where there are $L$ users and a single relay. Each transmitter sends a real-valued codeword, $\mathbf{x}_{l} \in \mathbb{R}^{n}$ with rate $R$, which is subject to a power constraint $P$. The relay observes a noisy linear combination of the transmitted signals through the channel,

$$
\mathbf{y}=\sum_{l=1}^{L} h_{l} \mathbf{x}_{l}+\mathbf{z}
$$

where $h_{l} \sim \mathcal{N}(0,1), 1 \leq l \leq L$, are the real channel coefficients and $\mathbf{z}$ is an i.i.d., Gaussian noise, $\mathbf{z} \sim \mathcal{N}(0, \mathbf{I})$. Let $\mathbf{h}_{L}=\left[h_{1}, h_{2}, \ldots, h_{L}\right]^{T}$ denote the vector of channel coefficients of all transmitting users. We assume that the relay knows the channel vector $\mathbf{h}_{L}$. In CF, after receiving the noisy linear combination, the relay selects an integer coefficients vector $\mathbf{a}=\left(a_{1}, a_{2}, \ldots, a_{L}\right)^{T} \in \mathbb{Z}^{L}$, and attempts to decode the lattice point $\sum_{l=1}^{L} a_{l} \mathbf{x}_{l}$ from $\mathbf{y}$. It then forward the decoded code-word towards the destination via a dedicated channel or another relay. The decoder, upon receiving enough such linear combinations of messages, decodes the original messages by solving the system of linear equations obtained from the coefficients vectors and the received code-words in each transmission phase. That is, successful decoding at the decoder is conditioned on the ability of the relay to decode the correct linear combination of messages and the full rank (i.e., rank $L$ ) of the matrix $\mathbf{A}$ which its raws are the coefficients vectors of each phase. Accordingly, we note that the number of transmission phases may be more than $L$.

The computation rate of the linear combination, with respect to the coefficients vector $\mathbf{a}$, is [1]:

$$
\mathcal{R}\left(\mathbf{h}_{L}, \mathbf{a}\right)=\frac{1}{2} \log ^{+}\left(\left(\|\mathbf{a}\|^{2}-\frac{P\left(\mathbf{h}_{L}^{T} \mathbf{a}\right)^{2}}{1+P\left\|\mathbf{h}_{L}\right\|^{2}}\right)^{-1}\right),
$$

where $\log ^{+}(x) \triangleq \max \{\log (x), 0\}$.

In order for the relay to decode a linear combination with a coefficients vector $\mathbf{a}$, all messages rates, for messages which have a non-zero value in their corresponding entry in a, must comply with the rate in (2). That is, $R<\mathcal{R}\left(\mathbf{h}_{L}, \mathbf{a}\right)$. Note that only messages with a non-zero entry in a are considered in the linear combination. We thus define as our performance measure the system's sum of computation rates to be the number of non-zero entries in a times $\mathcal{R}\left(\mathbf{h}_{L}, \mathbf{a}\right)$. This metric captures the computation rate of the relay along with the number of messages which are not considered as noise and take an active part in the decoding of the linear combination.

Since the relay can decide which linear combination to decode (i.e., to choose the coefficients vector $\mathbf{a}$ ), the relay can choose a which maximizes $\mathcal{R}\left(\mathbf{h}_{L}, \mathbf{a}\right)$ for a given $\mathbf{h}_{L}$. Note that according to [1. Lemma 1], the search domain for this maximizing a is restricted to all vectors $\mathbf{a}$ for which $\|\mathbf{a}\|^{2} \leq 1+P\left\|\mathbf{h}_{L}\right\|^{2}$. A polynomial time algorithm with complexity $O\left(L^{2} \sqrt{1+P\left\|\mathbf{h}_{L}\right\|^{2}}\right)$, which finds this maximizing a, was introduced in [8].
When the number of users $L$ is large, in [6] we showed that with probability that goes to 1 with $L$, the coefficients vector which will maximize $\mathcal{R}\left(\mathbf{h}_{L}, \mathbf{a}\right)$ is actually a unit vector. Specifically, [6] provides the following result.

Theorem 1 ( [6]). Under the CF scheme, the probability that a non-trivial vector a will be the coefficients vector which maximize the achievable rate $\mathcal{R}\left(\mathbf{h}_{L}, \mathbf{a}\right)$, compared to a unit vector $\mathbf{e}_{i}$, is upper bounded by

$$
\operatorname{Pr}\left(\mathcal{R}\left(\mathbf{h}_{L}, \mathbf{e}_{i}\right) \leq \mathcal{R}\left(\mathbf{h}_{L}, \mathbf{a}\right)\right) \leq e^{-L E(L)},
$$

where $\mathbf{a}$ is any integer vector that is not a unit vector and $E(L)=\left(1-\frac{3}{L}\right) \log \|\mathbf{a}\|$.

As a consequence, when the number of users is large, there is only a single user which the relay is interested in decoding. Thus, all other users are considered as noise, and due to the decoding process of $\mathrm{CF}$, each one contributes "self" noise, expressed by the approximation error of its channel gain to zero. This "self" noise degrades the achievable rate significantly, which eventually goes to zero as $L$ grows. Therefore, a restriction on the number of simultaneously transmitting users must be made in order to have a rate which does not go to zero. Thus, in this work, we examine such user scheduling in the context of CF schemes, devise an asymptotically optimal algorithm, and show that in this case the complex optimization on $\mathbf{a}$ itself is straightforward, resulting in a very efficient algorithm.

\section{SCHEDULING IN CF}

In this section, we present the specific scheduling problem of CF. Specifically, we do not describe the scheduling process itself, but rather center our interest on how the optimal schedule should be. We assume that in each transmission a subset of $k$ users are chosen by the scheduler. The total number of subsets is $\left(\begin{array}{l}L \\ k\end{array}\right)$, each having a channel vector which we denote by $\mathbf{h}$, and a corresponding optimal vector $\mathbf{a}$.

Definition 1 (The optimal schedule). The optimal schedule is a subset of $k$ users which yields the highest sum of computation rates. The sum-rate achieved by this schedule is

$$
\underset{\mathbf{h} \in \mathcal{H}^{\mathcal{S}}, \mathbf{a}}{\arg \max }\left\{\sum_{i=1}^{k} \mathbb{1}_{\left\{a_{i} \neq 0\right\}} \mathcal{R}(\mathbf{h}, \mathbf{a})\right\},
$$

where $\mathcal{H}^{\mathcal{S}}$ is the set of all vectors of length $k$ out of the channel vector $\mathbf{h}_{L}$.

Note that maximizing the sum-rate of a single transmission may not suffice to achieve this rate in the long-run, as one has to make sure these linear combinations indeed sum up to a full rank matrix. We will discusse these consideration in the sequel.

The scheduling problem consists of two highly connected optimization problems. The first can be viewed as finding the proper $\mathbf{h}$, and the second is finding the proper a (for that $\mathbf{h}$ ). A naive solution for this scheduling problem is to compute the sum-rate for all subsets of size $k$ (searching over 


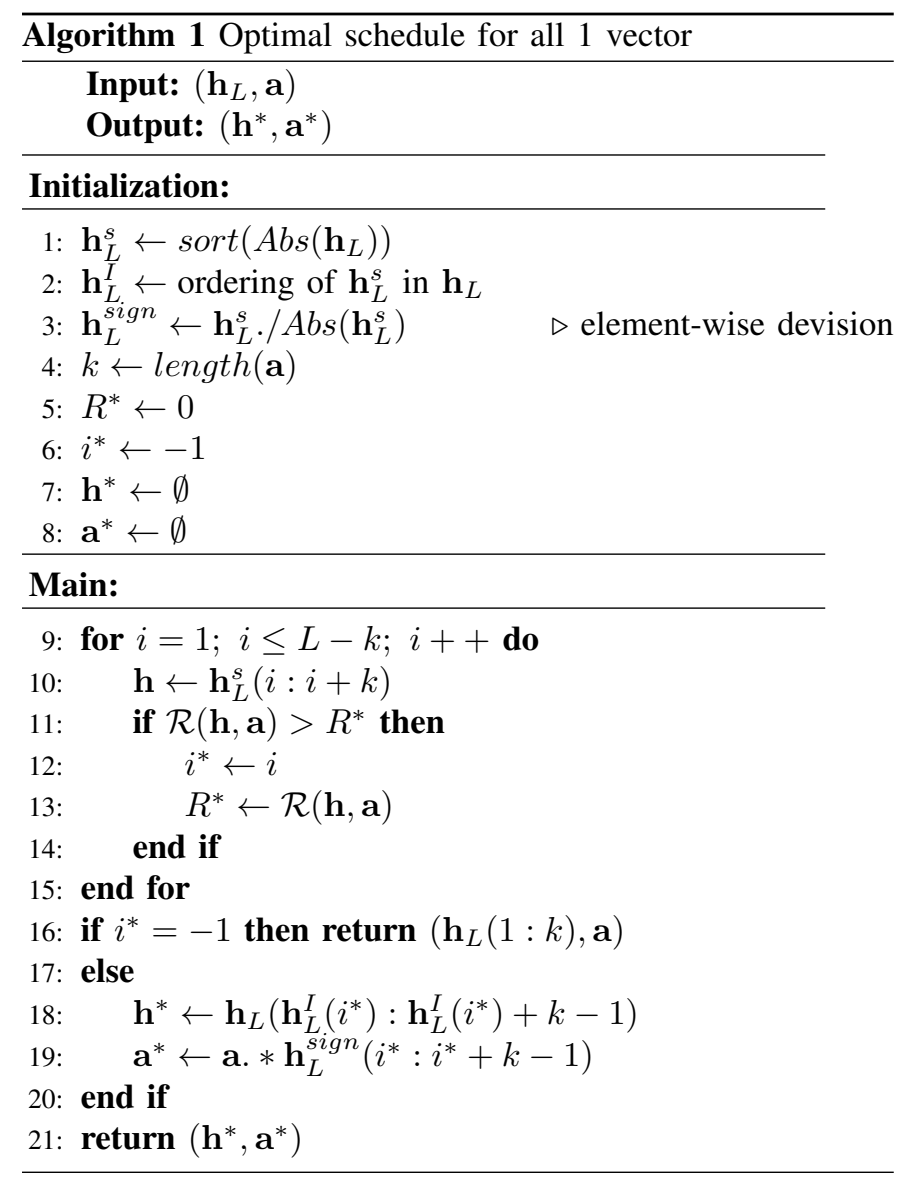

all $\mathbf{h}$ and the matching a) and choose the maximum among them. Since one has $\left(\begin{array}{l}L \\ k\end{array}\right) \approx L^{k}$ subsets, and for each subset there are $O\left(k^{2} \sqrt{1+P\left\|\mathbf{h}^{2}\right\|}\right)$ candidate coefficient vectors, the complexity is polynomial in $L$ but exponential in $k$. In fact, even for fixed $k$, such a complexity might be too high if $L$ is large. In this work, we provide a polynomial time (in both $k$ and $L$ ) scheduling algorithm that finds the asymptotically (with $L$ ) optimal schedule for any fixed $P$.

\section{A. Scheduling algorithm}

The scheduling algorithm seeks a subset of users which has a channel vector which best fits a fixed coefficients vector, $\mathbf{a}^{1} \triangleq\left(a_{1}, a_{2}, \ldots, a_{k}\right)$ such that $\left|a_{i}\right|=1, \forall i$. By this choice, the coefficients vector has non-zero entries and has the smallest norm value (out of all vectors with all non-zero entries), i.e., $\left\|\mathbf{a}^{\mathbf{1}}\right\|^{2}=k$. Note that this definition defines a set of $2^{k}$ coefficients vectors, denoted as $\mathbf{a}^{\{\mathbf{1}\}}$, which corresponds to the possible differences in the signs of the elements. In this case, since there are no zero entries, the sum-rate will be the achievable rate of the scheduled subset times $k$. Thus, the algorithm seeks the schedule which maximizes the sum-rate:

$$
k \cdot \max _{\mathbf{h} \in \mathcal{H}^{\mathcal{S}}}\left\{\max _{\mathbf{a} \in \mathbf{a}\{\mathbf{1}\}}\{\mathcal{R}(\mathbf{h}, \mathbf{a})\}\right\} .
$$

The following Lemma shows an important property of the optimal coefficients vector which maximizes the achievable rate.
Lemma 1. The optimal vector a satisfies either, $\operatorname{sign}\left(h_{i}\right)=$ $\operatorname{sign}\left(a_{i}\right)$ for all $i$ or $\operatorname{sign}\left(h_{i}\right) \neq \operatorname{sign}\left(a_{i}\right)$ for all $i$.

Proof: Considering the rate expression (2), since $\|\mathbf{a}\|^{2}$ does not depend on the signs, the optimal a must maximize the inner product $\left(\mathbf{h}^{T} \mathbf{a}\right)^{2}$. Obviously, all signs must match in order to have only positive elements in the summation of the inner product.

Lemma 1 implies that the inner maximization in (5) is trivial, since given a subset of channel coefficients $\mathbf{h} \in \mathcal{H}^{\mathcal{S}}$, the optimal $\mathbf{a} \in \mathbf{a}^{\{\mathbf{1}\}}$ is clear - just set the signs according to those of $\mathbf{h}$. Consequently, the following procedure is optimal for solving (5): disregard the signs in $\mathbf{h}_{L}$; find the optimal subset $\left(\left|h_{1}\right|,\left|h_{2}\right|, \ldots,\left|h_{k}\right|\right)$, a one which best fits $\mathbf{a}=(1,1, \ldots, 1) \triangleq \mathbf{1}$; then simply set the signs of a from all positive to the original signs of $\mathbf{h}$. This reduces the double optimization in (5), with $2^{k}$ options in the inner one, to a much simpler optimization:

$$
k \max _{\mathbf{h} \in \mathcal{H}^{\mathcal{S}}}\{\mathcal{R}(|\mathbf{h}|, \mathbf{1})\} .
$$

The following lemma shows that for the case of all-ones coefficients vector, this search can be simplified after sorting the channel vector $\mathbf{h}_{L}$ by the elements' absolute value. Thus, let us define $\mathbf{h}_{L}^{s}$ as $\left|\mathbf{h}_{L}\right|$ ordered in an ascending order.

Lemma 2. The optimal subset for the all-ones vector $\mathbf{1}$ is a subset of $k$ consecutive elements in $\mathbf{h}_{L}^{s}$. That is,

$$
\max _{\mathbf{h} \in \mathcal{H}^{\mathcal{S}}}\{\mathcal{R}(|\mathbf{h}|, \mathbf{1})\}=\max _{i}\left\{\mathcal{R}\left(\left|\mathbf{h}_{i}^{\prime}\right|, \mathbf{1}\right)\right\},
$$

where $\mathbf{h}_{i}^{\prime}=\left(h_{L, i}^{s}, \ldots, h_{L, i+k-1}^{s}\right)$ for $i \in[1, \ldots, L-k+1]$.

Proof: In order to show this property we refer to another expression for the achievable rate [1, Theorem 1],

$$
\mathcal{R}(\mathbf{h}, \mathbf{a})=\max _{\alpha \in \mathbb{R}} \frac{1}{2} \log ^{+}\left(\frac{P}{\alpha^{2}+P\|\alpha \mathbf{h}-\mathbf{a}\|^{2}}\right) .
$$

Note that if $\alpha=\frac{P \mathbf{h}^{T} \mathbf{a}}{1+P\|\mathbf{h}\|^{2}}$, i.e., the MMSE coefficient which maximizes the rate, we obtain the rate expression as presented in equation (2). However, for a general and fixed $\alpha$ we have,

$$
\begin{aligned}
& \max _{\mathbf{h} \in \mathcal{H}^{\mathcal{S}}}\{\mathcal{R}(|\mathbf{h}|, \mathbf{1})\} \\
& =\max _{\mathbf{h} \in \mathcal{H}^{\mathcal{S}}}\left\{\frac{1}{2} \log ^{+}\left(\frac{P}{\min _{\alpha \in \mathbb{R}}\left\{\alpha^{2}+P\|\alpha|\mathbf{h}|-\mathbf{1}\|^{2}\right\}}\right)\right\} \\
& =\frac{1}{2} \log ^{+}\left(\frac{P}{\min _{\mathbf{h} \in \mathcal{H}^{\mathcal{S}}}\left\{\min _{\alpha \in \mathbb{R}}\left\{\alpha^{2}+P\|\alpha|\mathbf{h}|-\mathbf{1}\|^{2}\right\}\right\}}\right) \\
& =\frac{1}{2} \log ^{+}\left(\frac{P}{\min _{\alpha>0}\left\{\min _{\mathbf{h} \in \mathcal{H}^{\mathcal{S}}}\left\{\alpha^{2}+P\|\alpha|\mathbf{h}|-\mathbf{1}\|^{2}\right\}\right.}\right)
\end{aligned}
$$

where in the last line we can reduce the minimization to $\alpha>$ 0 since for $\alpha<0$ we would increase the term for all $|\mathbf{h}|$. Therefore we need to show that for any $\alpha>0$

$$
\underset{\mathbf{h} \in \mathcal{H}^{\mathcal{S}}}{\arg \min }\left\{\|\alpha|\mathbf{h}|-\mathbf{1}\|^{2}\right\}=\mathbf{h}_{i}^{\prime},
$$




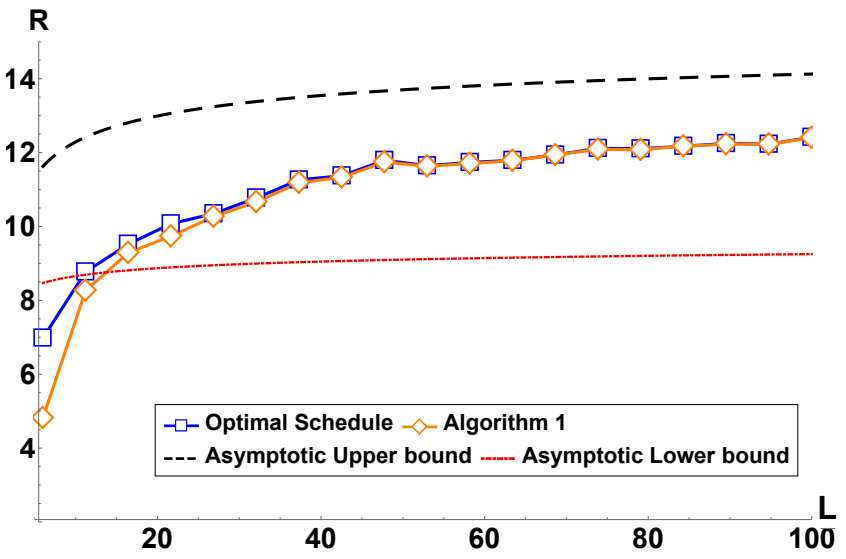

Fig. 1: The system's sum-rate of the scheduling algorithm compared with the optimal scheduled sum-rate for $k=3$, as a function of $L$ with $P=100$. The asymptotic lower and upper bound which was given in Theorems 3 and 4 , respectively, are also plotted. The lower bound was plotted with $\delta=0.005$.

for some $i \in[1, \ldots, L-k+1]$.

Let us define the sequence $\Delta_{j}=\left(\alpha h_{L, j}^{s}-1\right)^{2}$, for $j=$ $1, \ldots, L$. This sequence can be monotonic increasing, monotonic decreasing or monotonic decreasing and then monotonic increasing with $j$; it depends on the value of $\alpha h_{L, 1}^{s}$ and $\alpha h_{L, L}^{s}$ with respect to 1 . For example, if $\alpha h_{L, 1}^{s}>1$ then the sequence is monotonic increasing with $j$. Let us choose some $\mathbf{h} \in \mathcal{H}^{\mathcal{S}}$ such that its corresponding elements in $\mathbf{h}_{L}^{s}$ are not consecutive. Hence, w.l.o.g. assume that two elements in $\mathbf{h}$ corresponds to two elements $h_{L, i}^{s}$ and $h_{L, j}^{s}$ such that $i+1 \neq j$. Accordingly, either the choices $\mathbf{h}_{j}^{\prime}$ or $\mathbf{h}_{j-k}^{\prime}$ will minimize $\left\{\|\alpha|\mathbf{h}|-\mathbf{1}\|^{2}\right\}$ since in at least one of the choice we would decrease with the sequence $\Delta_{i}$. Note also that this is true for the choices $\mathbf{h}_{i}^{\prime}$ or $\mathbf{h}_{i-k}^{\prime}$

Considering Lemmas 1 and 2, the optimal algorithm for the optimization problem in (5) is presented as Algorithm 1. Accordingly, the complexity of the algorithm is $O((L-$ $k) L \log L)$ due to the sorting of $\mathbf{h}_{L}$ and the scan of $L-k$ scheduling options. The performance are summarized in the following theorem, whose proof is given in the sequel.

Theorem 2. Algorithm 1 attains the optimal scaling laws of the expected sum-rate, which is $O(\log \log L)$.

Theorem 2 implies that the choice of fixing the coefficients vector $\mathbf{a} \in \mathbf{a}^{\{\mathbf{1}\}}$ is asymptotically optimal as $L$ grows.

Simulation results of the system's expected sum-rate for Algorithm 1, compared with the optimal schedule (the naive solution) for $k=3$ as a function of $L$ are depicted Figure 1 . This simulation was compared also with the asymptotic upper and lower bounds in Theorems 3 and 4 below, which show good agreement even for moderate values of $L$.

\section{Sum-Rate Behaviour and the Scaling Law}

The proposed algorithm promises to find the optimal subset of users which attains the maximum sum-rate while fixing the coefficients vector a such that $\mathbf{a} \in \mathbf{a}^{\{\mathbf{1}\}}$. In this section, we start with a graphical interpretation for the problem of finding the optimal schedule; then, we provide a lower bound for Algorithm 1 and compare it to a global upper bound on the achievable rate [1]. Using it, we conclude that the scaling law of the suggested algorithm is similar to the best performance any scheduled subset can achieve with $\mathrm{CF}$, giving Theorem 2

\section{A. Achievable rate under scheduling}

We now provide a graphical interpretation for the problem of finding the optimal schedule. This interpretation is based on the analysis of an upper bound on the achievable rate, yet gives the motivation for the suggested algorithm. Specifically, it explains the reason for ignoring scheduling opportunities which attain insignificant improvement in the achievable rate.

Consider the achievable rate of a given schedule:

$$
\begin{aligned}
\mathcal{R}(\mathbf{h}, \mathbf{a}) & =\frac{1}{2} \log ^{+}\left(\left(\|\mathbf{a}\|^{2}-\frac{P\left(\mathbf{h}^{T} \mathbf{a}\right)^{2}}{1+P\|\mathbf{h}\|^{2}}\right)^{-1}\right) \\
& \longrightarrow \frac{1}{P} \log ^{+}\left(\left(\|\mathbf{a}\|^{2}-\frac{\left(\mathbf{h}^{T} \mathbf{a}\right)^{2}}{\|\mathbf{h}\|^{2}}\right)^{-1}\right) \\
& =\frac{1}{2} \log ^{+}\left(\left(\|\mathbf{a}\|^{2}-\|\mathbf{a}\|^{2} \cos ^{2}(\theta)\right)^{-1}\right) \\
& =\frac{1}{2} \log ^{+}\left(\left(\|\mathbf{a}\|^{2} \sin ^{2}(\theta)\right)^{-1}\right),
\end{aligned}
$$

where $\theta$ is the angle between $\mathbf{h}$ and its coefficients vector $\mathbf{a}$.

Figure 2 depicts the behaviour of the achievable rate as a function of $\theta$ and $\|\mathbf{a}\|^{2}$. The discrete lines represent simulation results for the achievable rate of each subset of size $k=3$, out of a specific realization of the channel vector $\mathbf{h}_{L} . P=1000$. The continuous curve is a graphic representation of equation (9).

The continuous curve is a bit misleading since, for one, $\mathbf{a}$ is an integer vector, hence, its squared norms takes only integer values. Second, for a certain $\|\mathbf{a}\|^{2}$ there are a finite possible choices of a, e.g., for $\|\mathbf{a}\|^{2}=5$ and dimension 2 the possible vectors are only $(1,2),(-1,2),(1,-2)$ and $(-1,-2)$. That is, in this case there are 4 possible angles for a given $\mathbf{h}$. Thus, this curve should look like a discrete plot. Yet, for ease of visualization and to recognize the rate behavior, we plotted a continuous curve. Note that the curve consider all integer vectors and not only the optimal for a certain $\mathbf{h}$.

There are several observations which can be inferred from Figure 2. The slope of the rate as a function of $\theta$ is much sharper than the slope of the rate as a function of $\|\mathbf{a}\|^{2}$, with an exception for the smallest values of $\|\mathbf{a}\|^{2}$ where a tip is noticeable. For the minimum possible value of $\|\mathbf{a}\|^{2}$, i.e. a unit vector, the rate is non zero for all angles in particular for small values of angels. This suggests that the rate is far more sensitive to small changes in the angle than small changes in $\|\mathbf{a}\|^{2}$. That is, for a given $\mathbf{h}$, if the angle between the coefficients vector and $\mathbf{h}$ is small, a high norm may be sustainable without much loss in optimality. On the other hand the opposite is not true. 


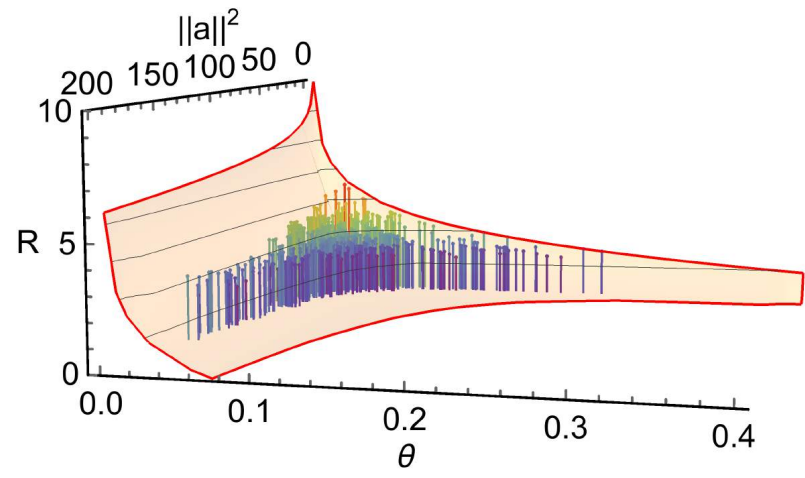

(a) $L=15$

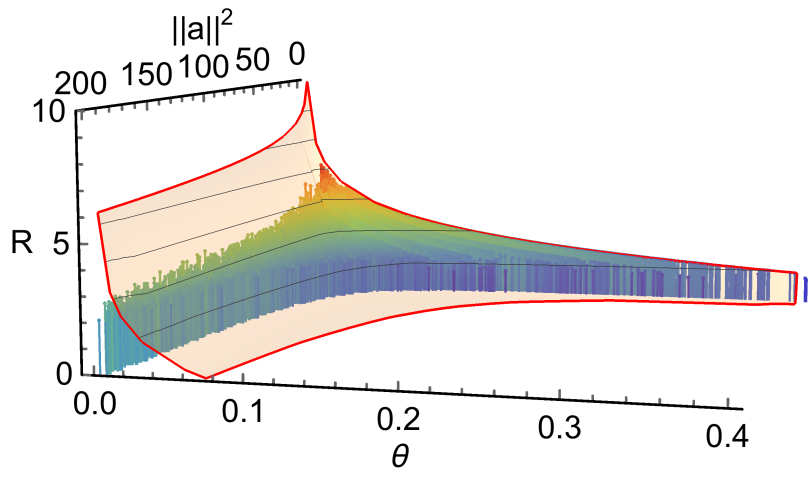

(b) $L=45$

Fig. 2: The achievable rate as a function of $\theta$, i.e., the angle between $\mathbf{a}$ and $\mathbf{h}$, and the squared norm of $\mathbf{a}$. The discrete points are simulation results for the rate of each subset of users of size $k=3$ for a specific realization of the channel vector $\mathbf{h}_{L}$ with $(a) L=15,(b)=L=45$ and $P=1000$. The transparent curved plane describes an upper bound on the rate as in (9), where, for ease of visualization, a continues function was plotted.

Another important observation is that, as the values of $\|\mathbf{a}\|^{2}$ grows, the slope is very small and thus we may only need to seek the scheduling solution in the dimension of $\theta$ without the risk of significant rate loss. In general we would still prefer $\|\mathbf{a}\|^{2}$ with low norm due to its penalty on the rate.

We emphasis that the assumption of $P \rightarrow \infty$ essentially means that the search domain becomes infinite. That is, for each given $\mathbf{h}$ we can find an excellent collinear integer approximation by considering vectors with very high norm in order to decrease the angle between the vectors. In this case, one should also consider the complexity of this search which is polynomial with $P$.

Figure 2 can be explained also in the following manner. In the rate maximization problem, for a certain $\mathbf{h}$, we have a sample (of points) out of the continuous curve in Figure 2 (since not all values of the angles are possible). The optimal rate is a single point out of this sample. While in the scheduling problem, each schedule is a different sample and the scheduler chooses the optimal point out of all the samples. Naturally, the optimal points for all possible schedules will be placed as close as possible to the boundaries and close to zero on the angle axis where the rate is high. One can imagine it as follows. For a given dimension and a fixed upper bound on the norm value we have a finite collection of possible a vectors with a certain $\|\mathbf{a}\|^{2}$ value. That is, as the number of $\mathbf{h}$ vectors grows, i.e. $L$ grows, we get a reacher plot, i.e., more points will be added to the graph as can be seen from figure $2 \mathrm{a}$ and $2 \mathrm{~b}$ respectively.

It is clear from Figure 2 that the highest rates are obtained for small norm values. Specifically, as $L$ grows ( $k$ is still fixed) the optimal coefficients vector that attains the highest achievable rate is the unit vector. This can also be explained as a consequence of [6. Theorem 1], which shows the superiority, in probability, of a unit vector with comparison to a certain non-trivial coefficients vector. For example, for $k=3$, the probability for a certain non-trivial vector to be chosen as the optimal one, comparing to a unit vector, is at most 0.3 .
However, other low norm vectors attain significant high rate as well. This is a significant factor in terms of the sumrate: a specific schedule may have low-norm (contains zeroes) coefficients vector, which gives high achievable rate, but its sum-rate may be small with respect to other vectors which have more non-zero entries.

Thus, in terms of the sum-rate, it may be beneficial to schedule groups which have no zero entries in their coefficients vectors (with relatively high achievable rate). Figure 3 depicts simulation results of the sum-rate of each subset of users of size $k=3$, for a specific realization of the channel vector $\mathbf{h}_{L}$. The right most hand curve corresponds to $\|\mathbf{a}\|^{2}=1$, i.e., a unit vector gives very low sum-rate due to the presence of the $k-1$ zeros. On the other hand, one can notice that the highest sum-rates are obtained for coefficients vector with $\|\mathbf{a}\|^{2}=3$, which is the smallest norm value with no zeros.

Consequently, in order to find the optimal schedule, we expect to use only a small set of fixed coefficients vectors, which have a small norm, with no zero entries as a good schedule. Thus, when searching for the optimal schedule, we flip the order in our optimization: we fix a reasonably good $\mathbf{a}$, and search for the best $\mathbf{h}$. As it turns out, this will be asymptotically optimal.

\section{B. Best channel for a fixed a}

The polynomial algorithm as given in [8] finds the optimal coefficients vector a for a given channel vector $\mathbf{h}$. We now consider the opposite case in which we fix a specific a and seek the optimal $\mathbf{h}$ (that is, the optimal subset of senders) which maximize the achievable rate. We have, 


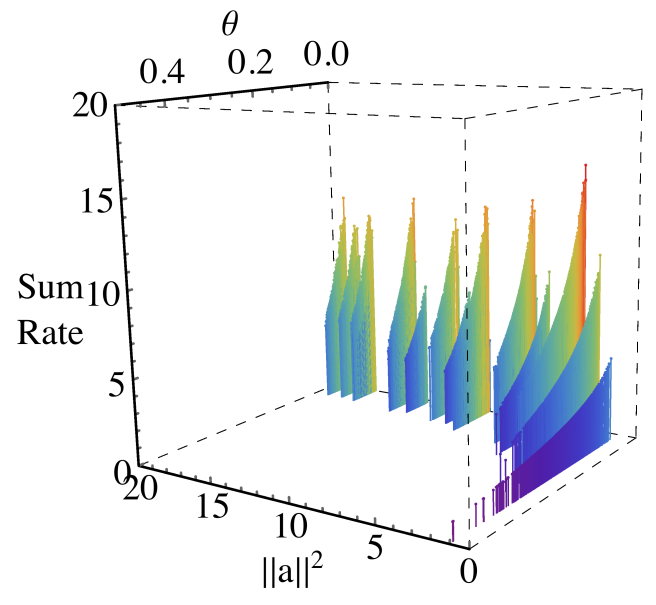

Fig. 3: The sum-rate of each subset of users as a function of the squared norm of the optimal a for the subset's channel vector and as a function of the angle between these vectors. Where $P=1000, L=45$ and $k=3$ for different number of users.

$$
\begin{aligned}
& \underset{\mathbf{h} \in \mathcal{H}^{\mathcal{S}}}{\arg \max }\{\mathcal{R}(\mathbf{h}, \mathbf{a})\} \\
& =\underset{\mathbf{h} \in \mathcal{H}^{\mathcal{S}}}{\arg \max }\left\{\frac{1}{2} \log ^{+}\left(\|\mathbf{a}\|^{2}-\frac{P\left(\mathbf{h}^{T} \mathbf{a}\right)^{2}}{1+P\|\mathbf{h}\|^{2}}\right)^{-1}\right\} \\
& =\frac{1}{2} \log ^{+}\left(\|\mathbf{a}\|^{2}-\underset{\mathbf{h} \in \mathcal{H}^{\mathcal{S}}}{\arg \max }\left\{\frac{P\left(\mathbf{h}^{T} \mathbf{a}\right)^{2}}{1+P\|\mathbf{h}\|^{2}}\right\}\right)^{-1} \\
& =\underset{\mathbf{h} \in \mathcal{H}^{\mathcal{S}}}{\arg \max }\left\{\frac{P\left(\mathbf{h}^{T} \mathbf{a}\right)^{2}}{1+P\|\mathbf{h}\|^{2}}\right\} \\
& =\underset{\mathbf{h} \in \mathcal{H}^{\mathcal{S}}}{\arg \max }\left\{\frac{P\|\mathbf{h}\|^{2}\|\mathbf{a}\|^{2} \cos ^{2}(\theta)}{1+P\|\mathbf{h}\|^{2}}\right\} \\
& =\underset{\mathbf{h} \in \mathcal{H}^{\mathcal{S}}}{\arg \max }\left\{\frac{\cos ^{2}(\theta)}{1+\frac{1}{P\|\mathbf{h}\|^{2}}}\right\} .
\end{aligned}
$$

Thus, the $\mathbf{h}$ which maximizes the achievable rate has a small angle with a and a high norm. However, this causes a tradeoff, since the highest norm vector may not be the one with the smallest angle to a. The scheduler should seek the optimal tradeoff point to maximize the achievable rate.

Considering the rate expression (10) for the regime of high SNR, i.e. $P \rightarrow \infty$, where we are left only with

$$
\underset{\mathbf{h} \in \mathcal{H}^{\mathcal{S}}}{\arg \max }\left\{\cos ^{2}(\theta)\right\}
$$

which essentially mean that the scheduler should only seek the group which has the smallest angle to a as the optimal scheduling policy. This can be seen in Figure 4 where we fixed various coefficients vectors and plotted the achievable rate for choosing the channel vector with the smallest angle comparing to the optimal choice (i.e. the schedule which gives the maximal rate) and a random choice. We note here that, for the case of $P \rightarrow \infty$, the scheduler eventually encounter the problem of choosing the maximum out of $\left(\begin{array}{l}L \\ k\end{array}\right)$ r.vs. which are distributed as $\operatorname{Beta}\left(\frac{1}{2}, \frac{k-1}{2}\right)$. We also note that some of these r.vs. are dependent due to the fact that $\mathcal{H}^{\mathcal{S}}$ is the set of all possible sub-sets of $\mathbf{h}_{L}$ which make it hard to analyse.

\section{Asymptotic guarantees}

We now give a lower bound on the performance of Algorithm 1 In particular, we show that asymptotically with $L$, the choice of an all-1 coefficients vector is optimal.

Theorem 3. The expected sum-rate of Algorithm 1 is lower bounded by the following,

$$
\begin{aligned}
& \mathbb{E}\left[k \max _{\mathbf{h} \in \mathcal{H}^{\mathcal{S}}}\{\mathcal{R}(|\mathbf{h}|, \mathbf{1})\}\right] \geq \\
& \frac{k}{2} \log ^{+}\left(k\left(1-\frac{P k u^{4}}{(u+\delta)^{2}\left(1+P k u^{2}\right)}(1-o(1))\right)\right)^{-1},
\end{aligned}
$$

where $u=\sqrt{2 \ln 2 \sqrt{L}}-\delta$ and $\delta$ is some small constant greater than zero.

Thus, the expected sum-rate for Algorithm 1 scales at least as $O\left(\frac{k}{4} \log \log L\right)$. o(1) $\rightarrow 0$ as $L \rightarrow \infty$.

The values $u$ and $\delta$ were chosen such that, with probability that goes to one with $L$, there are at least $k$ users with channel fading coefficients in the range $[u, u+\delta]$. Thus, we can lower bound the magnitude of the channel coefficients of the scheduled subset using $u$, and upper bound the angle between $\mathbf{h}$ and $\mathbf{1}$ using $\delta$. This bound applies (asymptotically with $L$ ) on the performance of Algorithm 1 since the best $k$ out of these users will be chosen. Theorem 3 indicates that indeed, as the number of users grows, the system's sum-rate grows as well, making scheduling not only mandatory but worthwhile. The proof tor Theorem 3 is given below.

In [1], the following universal upper bound for the achievable rate was given

$$
\mathcal{R}\left(\mathbf{h}, \mathbf{a}^{o p t}\right) \leq \frac{1}{2} \log \left(1+P \max _{i}\left\{h_{i}^{2}\right\}\right),
$$

where $\mathbf{h}$ is any channel vector of dimension $k$ and $\mathbf{a}^{o p t}$ is the coefficients vector which maximize the achievable rate. Using this result, one can derive an upper bound on the expected performance of any scheduling algorithm and its scaling laws, at the limit of large $L$.

Theorem 4. The expected sum-rate of any scheduling algorithm, is upper bounded by the following,

$$
\begin{gathered}
\mathbb{E}\left[k \max _{\mathbf{h} \in \mathcal{H}^{\mathcal{S}}}\left\{\mathcal{R}\left(\mathbf{h}, \mathbf{a}^{\text {opt }}\right)\right\}\right] \leq \\
\frac{k}{2} \log \left(1+P\left(2 \ln L-\ln \ln L-2 \ln \Gamma\left(\frac{1}{2}\right)+\frac{\gamma}{2}+o(1)\right)\right),
\end{gathered}
$$

where $\gamma$ is the Euler-Mascheroni constant.

Thus, the expected sum-rate for the suggested scheduling algorithm scales at most as $\frac{k}{2} \log \log L$. o(1) $\rightarrow 0$ as $L \rightarrow \infty$.

Theorems 3 and 4 show that the Algorithm 1 is asymptotically optimal as the upper and lower bounds on the performance scale as $O(\log \log L)$. This proves Theorem 2 . 


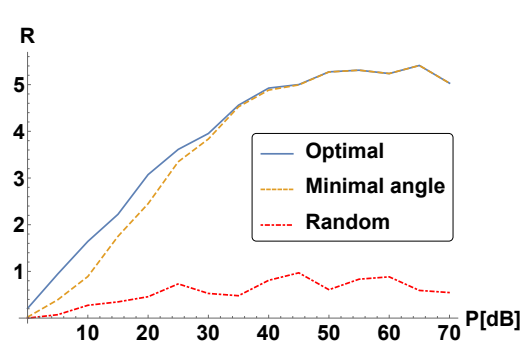

(a) $\mathbf{a}=(2,1,1)$

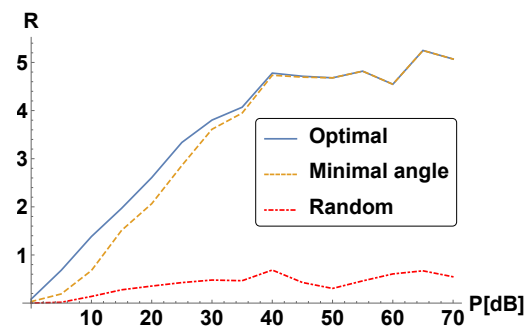

(b) $\mathbf{a}=(2,2,1)$

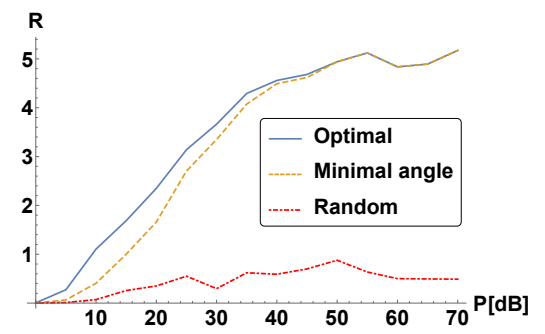

(c) $\mathbf{a}=(3,2,1)$

Fig. 4: Achievable rate for scheduling $k=3$ out of $L=20$ users with different fixed coefficients vectors. The users were chosen randomly (dot-dashed), optimally (solid), or according to the minimal angle between the channel vector of the scheduled group and $\mathbf{a}$.

Proof of Theorem 3 . We have,

$$
\begin{aligned}
& \mathbb{E}\left[k \max _{\mathbf{h} \in \mathcal{H}^{\mathcal{S}}}\{\mathcal{R}(|\mathbf{h}|, \mathbf{1})\}\right] \\
& =\mathbb{E}\left[k \max _{\mathbf{h} \in \mathcal{H}^{\mathcal{S}}}\left\{\frac{1}{2} \log ^{+}\left(k-\frac{P\left(|\mathbf{h}|^{T} \mathbf{1}\right)^{2}}{1+P\|\mathbf{h}\|^{2}}\right)\right\}\right] \\
& =\mathbb{E}\left[\frac{k}{2} \log ^{+}\left(k-\max _{\mathbf{h} \in \mathcal{H}^{\mathcal{S}}}\left\{\frac{P\left(|\mathbf{h}|^{T} \mathbf{1}\right)^{2}}{1+P\|\mathbf{h}\|^{2}}\right\}\right)^{-1}\right] \\
& \stackrel{(a)}{\geq} \mathbb{E}\left[\frac{k}{2} \log ^{+}\left(k-\frac{P\left(\mathbf{h}^{\prime T} \mathbf{1}\right)^{2}}{1+P\left\|\mathbf{h}^{\prime}\right\|^{2}}\right)^{-1}\right] \\
& \stackrel{(b)}{\geq} \frac{k}{2} \log ^{+}\left(k-\mathbb{E}\left[\frac{P\left(\mathbf{h}^{\prime T} \mathbf{1}\right)^{2}}{1+P\left\|\mathbf{h}^{\prime}\right\|^{2}}\right]\right)^{-1} \\
& =\frac{k}{2} \log ^{+}\left(k-\mathbb{E}\left[\frac{P k\left\|\mathbf{h}^{\prime}\right\|^{2} \cos ^{2}\left(\theta^{\prime}\right)}{1+P\left\|\mathbf{h}^{\prime}\right\|^{2}}\right]\right)^{-1},
\end{aligned}
$$

where in $(a)$ we chose some specific $\mathbf{h}^{\prime} \in \mathcal{H}^{\mathcal{S}}$ and $(b)$ follows from Jensen's inequality. As section IV-B suggests, the optimal schedule should be a subset of users with a high norm channel vector and a small angle between its channel vector and the corresponding coefficients vector. Thus, let us define the values $u(L)$ and $\delta$ such that $\mathbf{h}^{\prime}$ maintains $u \leq\left|h_{i}^{\prime}\right| \leq u+\delta, \forall i$. With this definition we are able to bound the parameters for a good schedule. With this definition, we are able to bound the parameters for a good schedule. The values of $u(L)$ and $\delta$ can help us tune the norm (by taking a high value of $u$ ) and the angle with 1 (by taking a small value of $\delta$ ) to attain a better bound as a function of $L$. And let us define $P_{r}(\xi)$ as the probability of having at least $k$ elements in $\mathbf{h}_{L}$ such that we can find an $\mathbf{h}^{\prime}$ satisfying the constraint above. We thus can write the last equation in (13) as follows,

$$
\begin{gathered}
=\frac{k}{2} \log ^{+}\left(k-\left(\mathbb{E}\left[\frac{P k\left\|\mathbf{h}^{\prime}\right\|^{2} \cos ^{2}\left(\theta^{\prime}\right)}{1+P\left\|\mathbf{h}^{\prime}\right\|^{2}} \mid \xi\right] P_{r}(\xi)+\right.\right. \\
\left.\left.\mathbb{E}\left[\frac{P k\left\|\mathbf{h}^{\prime}\right\|^{2} \cos ^{2}\left(\theta^{\prime}\right)}{1+P\left\|\mathbf{h}^{\prime}\right\|^{2}} \mid \bar{\xi}\right]\left(1-P_{r}(\xi)\right)\right)\right)^{-1} \\
\geq \frac{k}{2} \log ^{+}\left(k-\mathbb{E}\left[\frac{P k\left\|\mathbf{h}^{\prime}\right\|^{2} \cos ^{2}\left(\theta^{\prime}\right)}{1+P\left\|\mathbf{h}^{\prime}\right\|^{2}} \mid \xi\right] P_{r}(\xi)\right)^{-1} .
\end{gathered}
$$

Considering the conditioning we can lower bound $\left\|\mathbf{h}^{\prime}\right\|^{2}$ and $\cos ^{2}\left(\theta^{\prime}\right)$ as follows,

$$
\begin{aligned}
& \left\|\mathbf{h}^{\prime}\right\|^{2} \geq k u^{2} \\
& \cos ^{2}\left(\theta^{\prime}\right)=\frac{\left(\sum_{i=1}^{k} h_{i}^{\prime}\right)^{2}}{k\left\|\mathbf{h}^{\prime}\right\|^{2}} \geq \frac{k^{2} u^{2}}{k^{2}(u+\delta)^{2}}=\frac{1}{1+\frac{2 \delta}{u}+\frac{\delta^{2}}{u^{2}}} .
\end{aligned}
$$

The probability $P_{r}(\xi)$ can be computed using the binomial distribution with probability of success $p(u, \delta)=2(\Phi(u)-$ $\Phi(u+\delta))$ where $\Phi$ is the CDF of the normal distribution and can be lower bounded using the Chernoff bound. That is,

$$
\begin{aligned}
P_{r}(\xi) & =\sum_{i=k}^{L}\left(\begin{array}{c}
L \\
i
\end{array}\right) p(u, \delta)^{i}(1-p(u, \delta))^{L-i} \\
& =1-\sum_{i=0}^{k-1}\left(\begin{array}{c}
L \\
i
\end{array}\right) p(u, \delta)^{i}(1-p(u, \delta))^{L-i} \\
& \geq 1-e^{-\frac{1}{2 p(u, \delta)} \frac{(L p(u, \delta)-(k-1))^{2}}{L}} .
\end{aligned}
$$

Note that in order that $P_{r}(\xi)$ will go to one with $L, p(u, \delta)$ must decay at most as $\frac{1}{\sqrt{L}}$. Therefore, we would like to find $u$ and $\delta$ which will maintain this behaviour. That is, we wish to find $u$ and $\delta$ such that,

$$
\lim _{L \rightarrow \infty} \frac{p(u, \delta)}{1 / \sqrt{L}}=c,
$$


where $c \in(0, \infty]$. Thus,

$$
\begin{aligned}
& \lim _{L \rightarrow \infty} \frac{p(u, \delta)}{1 / \sqrt{L}} \\
& =\lim _{L \rightarrow \infty} \frac{2(\Phi(u)-\Phi(u+\delta))}{1 / \sqrt{L}} \\
& =\lim _{L \rightarrow \infty} \frac{\frac{1}{\sqrt{2 \pi}} \int_{u}^{u+\delta} e^{-\frac{t^{2}}{2}} d t}{1 / 2 \sqrt{L}} \\
& \stackrel{(a)}{\geq} \lim _{L \rightarrow \infty} \frac{\delta \frac{1}{\sqrt{2 \pi}} e^{-\frac{(u+\delta)^{2}}{2}}}{1 / 2 \sqrt{L}} \\
& \stackrel{(b)}{=} \lim _{L \rightarrow \infty} \frac{\delta \frac{1}{\sqrt{2 \pi}} e^{-\frac{\sqrt{2 \ln (2 \sqrt{L})^{2}}}{2}}}{1 / 2 \sqrt{L}} \\
& =\lim _{L \rightarrow \infty} \frac{\delta}{\sqrt{2 \pi}}
\end{aligned}
$$

Where in $(a)$ we bound the probability by the length of the interval and the density function smallest value in the interval $[u, u+\delta]$. Setting $u=\sqrt{2 \ln 2 \sqrt{L}}-\delta$ in (b) guarantees the desired outcome as long as $\delta$ is a constant grater than zero. Thus, for

$$
\begin{aligned}
p(u, \delta) & =\delta \frac{1}{\sqrt{2 \pi}} e^{-\frac{u^{2}}{2}} \\
& =\delta \frac{1}{\sqrt{2 \pi}} e^{-\frac{\sqrt{2 \ln (2 \sqrt{L})}}{2}} \\
& =\delta \frac{1}{\sqrt{2 \pi}} \frac{1}{2 \sqrt{L}}
\end{aligned}
$$

$P_{r}(\xi)$ will go to one with $L$. We note here that we require that $k-1<L p(u, \delta)$ for the correctness of the Chernoff bound in (16). That is, $k<\frac{\delta}{2 \sqrt{2 \pi}} \sqrt{L}+1$.

Setting (15) and (16) in (14) we get

$$
\begin{aligned}
& \frac{k}{2} \log ^{+}\left(k-\mathbb{E}\left[\frac{P k\left\|\mathbf{h}^{\prime}\right\|^{2} \cos ^{2}\left(\theta^{\prime}\right)}{1+P\left\|\mathbf{h}^{\prime}\right\|^{2}} \mid \xi\right] P_{r}(\xi)\right)^{-1} \\
& \geq \frac{k}{2} \log ^{+}\left(k \left(1-\frac{1}{1+\frac{2 \delta}{u}+\frac{\delta^{2}}{u^{2}}} \frac{1}{\frac{1}{P k u^{2}}+1}\right.\right. \\
& \quad \cdot\left(1-e^{\left.\left.\left.-\frac{1}{2 p(u, \delta)} \frac{(L p(u, \delta)-(k-1))^{2}}{L}\right)\right)\right)^{-1}}\right. \\
& \stackrel{(a)}{=} \frac{k}{2} \log ^{+}\left(k\left(1-\frac{1}{1+\frac{2 \delta}{u}+\frac{\delta^{2}}{u^{2}}} \frac{1}{\frac{1}{P k u^{2}}+1}(1-o(1))\right)\right)^{-1} \\
& \stackrel{(a)}{=} \frac{k}{2} \log ^{+}\left(k\left(1-\frac{P k u^{4}}{(u+\delta)^{2}\left(1+P k u^{2}\right)}(1-o(1))\right)\right)^{-1},
\end{aligned}
$$

where $(a)$ follows from the asymptotic behaviour of the exponent when setting $p(u, \delta)$ as in 19 . This can be seen as follows,

$$
\begin{aligned}
& \lim _{L \rightarrow \infty} \frac{e^{-\frac{1}{2 p(u, \delta)} \frac{(L p(u, \delta)-(k-1))^{2}}{L}}}{1} \\
& =\lim _{L \rightarrow \infty} e^{-\frac{1}{2 \delta \frac{1}{\sqrt{2 \pi}} \frac{1}{2 \sqrt{L}}} \frac{\left(L \delta \frac{1}{\sqrt{2 \pi}} \frac{1}{2 \sqrt{L}}-(k-1)\right)^{2}}{L}} \\
& =\lim _{L \rightarrow \infty} e^{-\frac{\sqrt{2 \pi} \sqrt{L}}{\delta} \frac{\left(\frac{\sqrt{L} \delta}{2 \sqrt{2 \pi}}-(k-1)\right)^{2}}{L}} \\
& =\lim _{L \rightarrow \infty} e^{-\frac{\sqrt{2 \pi} \sqrt{L}}{\delta}\left(\frac{\delta}{2 \sqrt{2 \pi}}-\frac{(k-1)}{\sqrt{L}}\right)^{2}} \\
& =e^{-\lim _{L \rightarrow \infty} \frac{\sqrt{2 \pi} \sqrt{L}}{\delta}\left(\frac{\delta}{2 \sqrt{2 \pi}}-\frac{(k-1)}{\sqrt{L}}\right)^{2}} \\
& =e^{-\infty}=0 .
\end{aligned}
$$

It can be verified (the computation mappears in Appendix A that the scaling laws of (20) indeed behave as $\frac{k}{4} \log \log L$ which completes the proof.

Proof of Theorem 44: Since the universal bound in (12) holds for all $\mathbf{h}$, it holds for any subset of users as well. Thus,

$$
\begin{aligned}
& \mathbb{E}\left[k \max _{\mathbf{h} \in \mathcal{H}^{\mathcal{S}}}\left\{\mathcal{R}\left(\mathbf{h}, \mathbf{a}^{o p t}\right)\right\}\right] \\
& \leq \mathbb{E}\left[k \max _{\mathbf{h} \in \mathcal{H}^{\mathcal{S}}}\left\{\frac{1}{2} \log \left(1+P \max _{i}\left\{h_{i}^{2}\right\}\right)\right\}\right] \\
& \stackrel{(a)}{=} \mathbb{E}\left[\frac{k}{2} \log \left(1+P \max _{i}\left\{h_{L i}^{2}\right\}\right)\right] \\
& \stackrel{(b)}{\leq} \frac{k}{2} \log \left(1+P \mathbb{E}\left[\max _{i}\left\{h_{L i}^{2}\right\}\right]\right) \\
& \stackrel{(c)}{=} \frac{k}{2} \log \left(1+P\left(2 \ln L-\ln \ln L-2 \ln \Gamma\left(\frac{1}{2}\right)+\frac{\gamma}{2}+o(1)\right)\right)
\end{aligned}
$$

where $(a)$ is true since the maximal element in $\mathbf{h}_{L}$ maximizes the expression and $(b)$ follows from Jensen's inequality. In (c) we used the asymptotic results for the expected value of the maximum value of a $\chi^{2}$ random vector of dimension $L$ in the limit of large $L$ [9. Table 3.4.4]. It can be verified (the computation appears in Appendix B) that the scaling laws indeed behave as $O\left(\frac{k}{2} \log \log L\right)$ which completes the proof.

\section{$D$. The value of $k$, completion time and future work}

Up until this point, the number of scheduled users $k$ is assumed as a fixed number. However, it may be optimized and dynamically changed in each transmission in order to provide addition gain to the overall performance of the system. This can be seen in the lower bound given in Theorem 3 where $k$ constitutes a pre-log factor for the system's sumrate. We emphasize that one cannot let $k$ be too large (at the order of $L$ ) and in fact it must satisfy $k<\frac{\delta}{2 \sqrt{2 \pi}} \sqrt{L}+1$ for the correctness of this bound. Additionally, one should also recall that Theorem 1 implicitly restrict the number of simultaneously transmitting users in order for the CF scheme be applicable.

Other possible improvement may be realized in the completion time of decoding all messages at the destination. As 
mentioned earlier, the coefficients vectors form the decoding matrix $\mathbf{A}$ of the linear system of equations to obtain the original $L$ messages. If one let $k$ users to transmit in each transmission phase, he essentially rules the sparseness of this matrix. Note that, although only $k$ users are scheduled for transmission in each phase, the decoding is done simultaneously for all messages so a coefficients vector (at the decoder) in each phase is of dimension $L$ and consist of the coefficients of the $k$ scheduled users and $L-k$ zeroes in the remaining entries. Accordingly, the following question may be asked. How many transmission phases required for complete decoding of all messages as a function of $k$. That is, how many linear combinations the destination must collect until $\mathbf{A}$ has rank $L$ (obviously, $L$ transmission phases must occur).

One can find resemblance to the known problem of coupons collector, where there are $L$ different coupons which are drawn randomly with replacement. Given this, how many draws are needed on average for the retrieval of all coupons. In our case, each coefficients vector can be considered as a coupon which is innovative or not. Namely, a new vector may increase the rank of the matrix formed by the collected vectors thus far, or it may be linearly dependent. For example, letting $k=1$ means that a single user is scheduled and thus each coefficients vector at the decoder is a unit vector. Since we have $L$ such unit vectors we get exactly the coupons collector problem which needs $O(L \log L)$ draws on average to obtain all coupons, i.e., $L$ independent coefficients vectors.

A different variation of the problem described above is considering the case where $k>1$ and in addition, assuming that the coefficients vectors are drawn randomly from the finite field $\mathbb{F}_{q}^{L}$ for $q>1$. If $k=L$, i.e., there in no restriction on the vectors, it is not hard to prove that the average number of vectors needed to obtain a matrix $\mathbf{A}$ with rank $L$ is $O(L)$, [10]. Specifically, even if $q=2$ the average number of transmission phases is at most $L+2$ [11].

Considering our scheduling problem, $k<<L$, and thus the received coefficients vectors are restricted to at least $L-k$ zero elements. In addition the elements of the vectors are in $\mathbb{Z}$. We would like to find the value of $k$ for which the average number of transmission phases is $O(L)$. Moreover, we would like to show that if we employ Algorithm 1, which ensure high rate for each linear combination by fixing the coefficients vector to be in $\mathbf{a}^{\{1\}}$, this average remains $O(L)$. We conjecture that in order to fulfil these requirements one needs $k=O(\log L)$.

\section{APPENDIX A}

PROOF FOR THE SCALING LAWS OF THEOREM 3

In order to prove that the scaling laws are $\frac{k}{4} \log \log L$ we will show that the limit of the division of the lower bound with $\frac{k}{4} \log \log L$ equals 1 as follows,

$$
\begin{aligned}
& \lim _{L \rightarrow \infty} \frac{\frac{k}{2} \log ^{+}\left(k\left(1-\frac{P k u^{4}}{(u+\delta)^{2}\left(1+P k u^{2}\right)}(1-g(L))\right)\right)^{-1}}{\frac{k}{4} \log \log L} \\
& =\lim _{L \rightarrow \infty} \frac{-2 \log ^{+}\left(k\left(1-\frac{P k u^{4}(1-g(L))}{(u+\delta)^{2}\left(1+P k u^{2}\right)}\right)\right)}{\log \log L} \\
& =0+\lim _{L \rightarrow \infty} \frac{-2 \log ^{+}\left(1-\frac{P k u^{4}(1-g(L))}{(u+\delta)^{2}\left(1+P k u^{2}\right)}\right)}{\log \log L}
\end{aligned}
$$

where $g(L)=e^{-\frac{1}{2 p(u, \delta)} \frac{(L p(u, \delta)-(k-1))^{2}}{L}}$ which we expressed as $o(1)$ in the theorem. We now lower and upper bound this limit to show that both bounds goes to one. Let us start with the upper bound.

$$
\begin{aligned}
& =\lim _{L \rightarrow \infty} \frac{-2 \log ^{+}\left(1-\frac{P k u^{4}(1-g(L))}{(u+\delta)^{2}\left(1+P k u^{2}\right)}\right)}{\log \log L} \\
& \leq \lim _{L \rightarrow \infty} \frac{-2 \log ^{+}\left(1-\frac{P k u^{4}}{(u+\delta)^{2}\left(1+P k u^{2}\right)}\right)}{\log \log L} \\
& \leq \lim _{L \rightarrow \infty} \frac{-2 \log ^{+}\left(1-\frac{u^{2}}{(u+\delta)^{2}}\right)}{\log \log L} \\
& \leq \lim _{L \rightarrow \infty} \frac{-2 \log \left(\frac{2 \delta u+\delta^{2}}{(u+\delta)^{2}}\right)}{\log \log L} \\
& \leq \lim _{L \rightarrow \infty} \frac{-2 \log \left(\frac{\delta u+\delta^{2}}{(u+\delta)^{2}}\right)}{\log \log L} \\
& \stackrel{(a)}{=} \lim _{L \rightarrow \infty} \frac{-2 \log +\left(\frac{\delta \sqrt{2 \log 2 \sqrt{L}}}{2 \log 2 \sqrt{L}}\right)}{\log \log L} \\
& =\lim _{L \rightarrow \infty} \frac{\log (2 \log 2 \sqrt{L})}{\log \log L} \\
& =\lim _{L \rightarrow \infty} \frac{\log \log 4 L}{\log \log L}=1 .
\end{aligned}
$$

In $(a)$ we set $u=\sqrt{2 \ln 2 \sqrt{L}}-\delta$. The lower bound is as follows,

$$
\begin{aligned}
& =\lim _{L \rightarrow \infty} \frac{-2 \log ^{+}\left(1-\frac{P k u^{4}(1-g(L))}{(u+\delta)^{2}\left(1+P k u^{2}\right)}\right)}{\log \log L} \\
& \geq \lim _{L \rightarrow \infty} \frac{-2 \log ^{+}\left(1-\frac{u^{4}(1-g(L))}{(u+\delta)^{2}\left(1+u^{2}\right)}\right)}{\log \log L} \\
& =\lim _{L \rightarrow \infty} \frac{2 \log ^{+}\left(\frac{(u+\delta)^{2}\left(1+u^{2}\right)}{(u+\delta)^{2}\left(1+u^{2}\right)-u^{4}(1-g(L))}\right)}{\log \log L} \\
& \geq \lim _{L \rightarrow \infty} \frac{2 \log ^{+}\left(\frac{(u+\delta)^{2} u^{2}}{(u+\delta)^{2}+2 u^{3} \delta+u^{2} \delta^{2}+u^{4} g(L)}\right)}{\log \log L} \\
& \geq \lim _{L \rightarrow \infty} \frac{2 \log ^{+}\left(\frac{(u+\delta)^{2} u^{2}}{(u+\delta)^{2}+2(u+\delta)^{3} \delta+(u+\delta)^{2} \delta^{2}+(u+\delta)^{4} g(L)}\right)}{\log \log L} \\
& =\lim _{L \rightarrow \infty} \frac{2 \log ^{+}\left(\frac{u^{2}}{1+2(u+\delta) \delta+\delta^{2}+(u+\delta)^{2} g(L)}\right)}{\log \log L} \\
& \stackrel{(a)}{=} \lim _{L \rightarrow \infty} \frac{\left(\sqrt{2 \ln 2 \sqrt{L}-\delta)^{2}}\right.}{2 \log ^{+}\left(\frac{\log \log L}{1+2 \delta \sqrt{2 \ln 2 \sqrt{L}+\delta^{2}+g(L) 2 \ln 2 \sqrt{L}}}\right)}
\end{aligned}
$$




$$
\begin{aligned}
& \geq \lim _{L \rightarrow \infty} \frac{2 \log ^{+}\left(\frac{2 \ln 2 \sqrt{L}-2 \delta \sqrt{2 \ln 2 \sqrt{L}}}{1+2 \delta \sqrt{2 \ln 2 \sqrt{L}}+\delta^{2}+g(L) 2 \ln 2 \sqrt{L}}\right)}{\log \log L} \\
& =\lim _{L \rightarrow \infty} \frac{2 \log ^{+}(\sqrt{2 \ln 2 \sqrt{L}}-2 \delta)}{\log \log L} \\
& -\lim _{L \rightarrow \infty} \frac{2 \log ^{+}\left(\frac{1+\delta^{2}}{\sqrt{2 \ln 2 \sqrt{L}}}+2 \delta+g(L) \sqrt{2 \ln 2 \sqrt{L}}\right)}{\log \log L} \\
& \geq \lim _{L \rightarrow \infty} \frac{\log ^{+}(2 \ln 2 \sqrt{L}-2 \delta \sqrt{2 \ln 2 \sqrt{L}})}{\log \log L} \\
& -\lim _{L \rightarrow \infty} \frac{2 \log ^{+}\left(\frac{2 \delta^{2}}{\sqrt{2 \ln 2 \sqrt{L}}}+2 \delta+g(L) 2 \ln 2 \sqrt{L}\right)}{\log \log L} \\
& \geq \lim _{L \rightarrow \infty} \frac{\log ^{+}(2 \ln 2 \sqrt{L}-2 \delta 2 \ln 2 \sqrt{L})}{\log \log L} \\
& -\lim _{L \rightarrow \infty} \frac{2 \log ^{+}\left(\delta^{2}+\delta+g(L) \ln 2 \sqrt{L}\right)}{\log \log L} \\
& \geq \lim _{L \rightarrow \infty} \frac{\log ^{+}(\ln 2 \sqrt{L})}{\log \log L} \\
& -\lim _{L \rightarrow \infty} \frac{2 \log ^{+}\left(\left(\delta^{2}+\delta+1\right)(g(L) \ln 2 \sqrt{L}+1)\right)}{\log \log L} \\
& =1-\lim _{L \rightarrow \infty} \frac{2 \log ^{+}(1+g(L) \ln 2 \sqrt{L})}{\log \log L} \\
& \geq 1-\lim _{L \rightarrow \infty} \frac{2 \log ^{+}(1+g(L) L)}{\log \log L} \\
& \stackrel{(b)}{=} 1-\lim _{L \rightarrow \infty} \frac{2 \log ^{+}\left(1+e^{-\frac{\sqrt{2 \pi} \sqrt{L}}{\delta}\left(\frac{\delta}{2 \sqrt{2 \pi}}-\frac{(k-1)}{\sqrt{L}}\right)^{2}} L\right)}{\log \log L} \\
& =1-\lim _{L \rightarrow \infty} \frac{2 \log ^{+}\left(1+e^{-\frac{\sqrt{L} \delta}{4 \sqrt{2 \pi}}} e^{(k-1)} e^{-\frac{\sqrt{2 \pi}(k-1)^{2}}{\delta \sqrt{L}}} L\right)}{\log \log L} \\
& \geq 1-\lim _{L \rightarrow \infty} \frac{2 \log ^{+}\left(1+e^{-\frac{\sqrt{L} \delta}{4 \sqrt{2 \pi}}} e^{(k-1)} L\right)}{\log \log L} \\
& =1-\lim _{L \rightarrow \infty} \frac{2 \log ^{+}\left(1+e^{-\frac{\sqrt{L} \delta}{4 \sqrt{2 \pi}}} L\right)}{\log \log L} \\
& \stackrel{(c)}{=} 1-2 \lim _{L \rightarrow \infty} \frac{\left(2-\frac{\delta}{4 \sqrt{2 \pi}} \sqrt{L}\right) L \log L}{2\left(e^{\frac{\sqrt{L} \delta}{4 \sqrt{2 \pi}}}+L\right)} \\
& =1
\end{aligned}
$$

In $(a)$ we set $u=\sqrt{2 \ln 2 \sqrt{L}}-\delta$, in $(b)$ we set $g(L)$ with its expression with $p(u, \delta)$ as in (19) and in $(c)$ we used L'Hospital's rule which completes the proof.

\section{APPENDIX B}

\section{PROOF FOR THE SCALING LAWS OF THEOREM 4}

In order to prove that the scaling laws are $\frac{k}{2} \log \log L$ we will show that the limit of the division of the lower bound with $\frac{k}{2} \log \log L$ equals 1 as follows,

$$
\begin{aligned}
& \lim _{L \rightarrow \infty} \frac{\frac{k}{2} \log \left(1+P\left(2 \ln L-\ln \ln L-2 \ln \Gamma\left(\frac{1}{2}\right)+\frac{\gamma}{2}\right)\right)}{\frac{k}{2} \log \log L} \\
& \lim _{L \rightarrow \infty} \frac{\log \left(1+P\left(2 \ln L-\ln \ln L-2 \ln \Gamma\left(\frac{1}{2}\right)+\frac{\gamma}{2}\right)\right)}{\log \log L}
\end{aligned}
$$

We now lower and upper bound this limit to show that both bounds goes to one. Let us start with the upper bound.

$$
\begin{aligned}
& \lim _{L \rightarrow \infty} \frac{\log \left(1+P\left(2 \ln L-\ln \ln L-2 \ln \Gamma\left(\frac{1}{2}\right)+\frac{\gamma}{2}\right)\right)}{\log \log L} \\
& \leq \lim _{L \rightarrow \infty} \frac{\log \left(P+P\left(2 \ln L-\ln \ln L-2 \ln \Gamma\left(\frac{1}{2}\right)+\frac{\gamma}{2}\right)\right)}{\log \log L} \\
& \leq \lim _{L \rightarrow \infty} \frac{\log \left(P\left(1+2 \ln L-\ln \ln L-2 \ln \Gamma\left(\frac{1}{2}\right)+\frac{\gamma}{2}\right)\right)}{\log \log L} \\
& \leq \lim _{L \rightarrow \infty} \frac{\log \left(1+2 \ln L-\ln \ln L-2 \ln \Gamma\left(\frac{1}{2}\right)+\frac{\gamma}{2}\right)}{\log \log L} \\
& \leq \lim _{L \rightarrow \infty} \frac{\log \left(1+2 \ln L+\frac{\gamma}{2}\right)}{\log \log L}=1 .
\end{aligned}
$$

The lower bound is as follows,

$$
\begin{aligned}
& \lim _{L \rightarrow \infty} \frac{\log \left(1+P\left(2 \ln L-\ln \ln L-2 \ln \Gamma\left(\frac{1}{2}\right)+\frac{\gamma}{2}\right)\right)}{\log \log L} \\
& \geq \lim _{L \rightarrow \infty} \frac{\log \left(2 \ln L-\ln \ln L-2 \ln \Gamma\left(\frac{1}{2}\right)+\frac{\gamma}{2}\right)}{\log \log L} \\
& \geq \lim _{L \rightarrow \infty} \frac{\log \left(2 \ln \ln L-\ln \ln L-2 \ln \Gamma\left(\frac{1}{2}\right)+\frac{\gamma}{2}\right)}{\log \log L} \\
& \geq \lim _{L \rightarrow \infty} \frac{\log \left(\ln \ln L-2 \ln \Gamma\left(\frac{1}{2}\right)\right)}{\log \log L}=1 .
\end{aligned}
$$

\section{REFERENCES}

[1] B. Nazer and M. Gastpar, "Compute-and-forward: Harnessing interference through structured codes," IEEE Transactions on Information Theory, vol. 57, no. 10, pp. 6463-6486, 2011.

[2] J. Zhan, B. Nazer, U. Erez, and M. Gastpar, "Integer-forcing linear receivers," IEEE Transactions on Information Theory, vol. 60, no. 12, pp. 7661-7685, 2014.

[3] O. Ordentlich, U. Erez, and B. Nazer, "The approximate sum capacity of the symmetric gaussian-user interference channel," IEEE Transactions on Information Theory, vol. 60, no. 6, pp. 3450-3482, 2014.

[4] L. Wei and W. Chen, "Compute-and-forward network coding design over multi-source multi-relay channels," IEEE Transactions on Wireless Communications, vol. 11, no. 9, pp. 3348-3357, 2012.

[5] S.-N. Hong and G. Caire, "Compute-and-forward strategies for cooperative distributed antenna systems," Information Theory, IEEE Transactions on, vol. 59, no. 9, pp. 5227-5243, 2013.

[6] O. Shmuel, A. Cohen, and O. Gurewitz, "The necessity of scheduling in compute-and-forward," in 2017 IEEE Information Theory Workshop (ITW), Nov 2017, pp. 509-513.

[7] D. Ramirez and B. Aazhang, "Scheduling for compute and forward networks," in 2015 49th Asilomar Conference on Signals, Systems and Computers. IEEE, 2015, pp. 57-58.

[8] S. Sahraei and M. Gastpar, "Compute-and-forward: Finding the best equation," in Communication, Control, and Computing (Allerton), 2014 52nd Annual Allerton Conference on. IEEE, 2014, pp. 227-233.

[9] P. Embrechts, C. Klüppelberg, and T. Mikosch, Modelling extremal events: for insurance and finance. Springer Science \& Business Media, 2013, vol. 33.

[10] A. Eryilmaz, A. Ozdaglar, and M. Medard, "On delay performance gains from network coding," in Information Sciences and Systems, 2006 40th Annual Conference on. IEEE, 2006, pp. 864-870.

[11] D. E. Lucani, M. Médard, and M. Stojanovic, "Random linear network coding for time-division duplexing: Field size considerations," in Global Telecommunications Conference, 2009. GLOBECOM 2009. IEEE. IEEE, 2009, pp. 1-6. 